ORIGINAL ARTICLE

\title{
A combined HPLC-immunoenzymatic comprehensive screening for suspected drug poisoning in the emergency department
}

\author{
A Fabbri, S Ruggeri, G Marchesini, A Vandelli
}

Emerg Med J 2004;21:317-322. doi: 10.1136/emj.2003.012344

See end of article for authors' affiliations

.....................

Correspondence to:

Dr A Fabbri, Dipartimento Emergenza-Urgenza

Accettazione, Ospedale

GB Morgagni, Azienda

USL di Forli, 1, P.le Solieri,

1-47100 Forli, Italy:

andfabbri@libero.it

Accepted for publication 30 October 2003
Objective: To review the results of a comprehensive drug screening as first line diagnostic tool in patients attending an emergency department for suspected drug poisoning.

Methods: A comprehensive drug screening was carried out in plasma or urine, or both, of 310 patients combining an HPLC multidrug profiling system and a fluorescence polarisation immunoassay.

Results: In $64.2 \%$ of cases the screening confirmed the diagnosis of drug poisoning, in $13.9 \%$ suspected drugs were measurable at non-toxic concentrations, and in $21.9 \%$ no drugs were found. The suspected drugs were fully confirmed in a minority of cases, (symptomatic patients: $28.2 \%$ compared with asymptomatic: $16.5 \%)$. Symptomatic patients were less likely to have at least one suspected drug $(29.6 \%$ compared with $57.7 \% ; \mathrm{p}<0.001$ ), and more likely to have at least one unsuspected drug found at analysis (17.4\% compared with $3.1 \% ; p=0.005)$. In $5 \%$ of patients, asymptomatic when first observed, one or more unsuspected drugs were found. In 6 of 29 patients, with suspected poisoning of an unspecified drug, the screening identified the specific drug and excluded acute intoxication in the remaining cases.

Conclusion: A rapid comprehensive drug screening adds to the diagnosis of patients with suspected drug poisoning, identifying unsuspected drugs in symptomatic patients and excluding drugs in asymptomatic subjects.
$\mathrm{T}$ he role of the emergency physician in the diagnosis of acute multidrug poisoning is important in preventing serious morbidity and mortality. Prior studies have reported discordance between clinical suspicion and laboratory test results in suspected drug overdoses. ${ }^{12}$ A comparison of the results of laboratory findings with a clinical judgment based on history, symptoms, and signs, showed that laboratory results may provide additional information as to the nature of the intoxication in about two thirds of cases. ${ }^{3} \mathrm{~A}$ new comprehensive screening for suspected drug poisoning has been proposed. ${ }^{4}$ Its usefulness has however been questioned $^{5}$; in most cases the results do not change treatment, the decision being mainly based on clinical parameters. ${ }^{6}$ On this basis, a limited drug screening to prove the presence of life threatening drugs is actually considered a more cost effective and efficient diagnostic tool. ${ }^{78}$

Comprehensive drug screening might be useful in patients with suspected poisoning. A pure clinical approach, without any confirmatory laboratory result, might be insufficient in deciding patients' treatment and disposition. In the presence of suspected drug poisoning, a few patients with minor symptoms may only need admission to an ordinary medical ward for minimal observation, instead of intensive care units. At the same time, patients with a negative screening might be safely discharged within a few hours, avoiding unnecessary in-hospital observation and potentially harmful gastrointestinal decontamination procedures.

However, caution is needed in patients with some types of poisoning where plasma concentrations can rise to potentially toxic levels after a negative drug screen.

We report the results obtained from a series of consecutive patients attending an emergency department (ED) for suspected drug poisoning. The information given by laboratory findings was compared with clinical data.

\section{METHODS \\ Patients}

We retrospectively examined all consecutive cases attending the ED of Forlì from December 1996 to May 2000, and who had a comprehensive drug screening because of suspected intentional drug poisoning or acute intoxication from drugs of misuse. According to the protocol in use in our ED, all subjects were submitted to a combined HPLC-immunoenzymatic screening for drug poisoning. Information gathered from patients' records included personal data, poisoning to admission time and type of suspected $\operatorname{drug}(\mathrm{s})$, presence of pre-existing chronic illnesses, main characteristics and symptoms of clinical presentation. These patients account for about $75 \%$ of all cases admitted for suspected drug poisoning in our institution, after exclusion of chemicals and carbon monoxide intoxication.

The suspicion of intentional drug poisoning was based on history, as reported by the patients, relatives, friends or bystanders, or by the finding of empty medicine containers, symptoms, and specific clinical signs of drug intoxication.

The clinical records of 310 subjects, median age 32 years, admitted to the ED with suspected drug overdose were reviewed. In these patients a total of 443 drugs were suspected. Table 1 shows their main clinical features. One hundred and nine cases $(35.2 \%)$ had concomitant intentional self harm injuries, 26 cases $(8.4 \%)$ had been involved in a road traffic accident, 8 cases $(2.6 \%)$ had medico-legal issues, and $4(1.3 \%)$ had traumatic injuries. Thirty nine cases $(12.6 \%)$ had coexisting medical diseases, and 196 subjects (63.2\%) were regularly treated with one or more drugs. Physicians suspected the involvement of specific drugs through a history obtained from the patients in 145 cases $(46.8 \%)$, from bystanders in 113 cases $(36.5 \%)$, through the finding of drug blisters or containers in 84 cases $(27.1 \%)$. In 
$29(9.4 \%)$ cases a drug screen was performed because of a moderate central nervous system depression, in the presence of unspecified drug poisoning. Most patients with central nervous system depression (249 cases) also had alcohol measured in blood. A blood alcohol concentration above the cut off value of $10 \mathrm{~g} / \mathrm{l}$ was present in 32 of 170 cases with acute drug overdose ( $18.8 \%$ ), and in 28 of 79 with no or trace amounts of drugs in blood (35.4\%).

A total of 101 patients (32.6\%) were discharged home after an observation period of three to six hours in the ED, 173 cases $(55.8 \%)$ were admitted to a general ward, 33 patients $(10.7 \%)$ to an intensive care unit. Three cases died within a few hours after admission to emergency room for morphine, lignocaine (lidocaine) overdose, and clomipramine plus clotiapine and cocaine overdose. Four other patients died after hospitalisation for subarachnoidal haemorrhage after cocaine intoxication, multiorgan failure after morphine, terfenadine, clotiapine and verapamil intoxication, and for untreatable ventricular fibrillation after clomipramine overdose.

\section{Drug screening}

The drug screening was performed by combining in sequence a multi-column HPLC drug profiling system (Remedi HS BioRad, Hercules, CA) and a fluorescence polarisation immunoassay (FPIA) with a TDx analyser (Abbott, Abbott Park, IL). Our protocol included the Remedi urinary drug screening and, in the case of positive drug detection, a confirmatory test with either Remedi in plasma or a quantitative FPIA, whenever available. ${ }^{9-12}$ FPIA was carried out in plasma for drugs and in urine samples for drugs of misuse (opioids, cannabis, amphetamines, cocaine).

Our current library of Remedi HS multidrug profiling system permits the determination of over 900 drugs and their metabolites of many common pharmaceutical agents (tables 2 and 3) that might be missed by limited screens routinely used in ED, within a detection limit for each substance of $200 \mathrm{ng} /$ ml. ${ }^{9-12}$ This policy guarantees a final result in a turn around time of 20-45 minutes. The system does not measure lithium, sulfonylureas, and any chemical agent. The FPIA system permits the quantitative determination of plasma benzodiazepines (BZD), digoxin, barbiturates, phenjtoin, methotrexate, primidone, salycilates, paracetamol, theophylline, tricyclic antidepressant (TCA), valproate, ethosuximide, and the urinary detection of the commonest drugs of misuse (opioids, cannabis, cocaine, amphetamines). To allow the clinician to correctly interpret the results of the screening, the problems and quality of results were always discussed with the analyst both before and after the test.

The combined analysis with Remedi in urine samples and plasma was performed in 116 cases (37.4\%). After the urinary Remedi screening, a quantitative confirmatory test with a single FPIA in plasma was necessary in 108 cases $(34.8 \%)$, whereas more than one test was performed in 33 cases (10.7\%). In the presence of drugs of misuse, a single confirmatory FPIA was carried out in urine in 55 cases $(17.7 \%)$ and more than one test in 12 cases $(4.5 \%)$ (table 2 ). The results reported thus represent the data generated through the usual clinical activity and physician judgment, and not a systematic use of all tests for every single case.

The diagnosis of drug poisoning and the evaluation of its severity were based on laboratory confirmation as well as on overall clinical assessment. Concordance and discordance of results between suspected drug poisoning and diagnosis was divided into four categories:

- laboratory test confirming precisely all suspected drugs;

- at least one suspected drug not confirmed at diagnosis;

- one clinically unsuspected drug reported at diagnosis;

- at least one suspected drug not confirmed and one suspected drug reported.

The protocol was carried out according to the Helsinki Declaration and approved by the local ethical committee of the Morgagni Hospital, Forlì.

\section{Data analysis}

Observed frequencies were used to describe data distribution. Odds ratio (OR) and 95\% confidence intervals (95\% CI) were also calculated. Statistical analyses were performed running the Stat-View 5.0 statistical package (SAS Institute, Cary, $\mathrm{NC)}$ on a personal computer. A two tailed $\mathrm{p}$ value less than 0.05 was considered for statistical significance.

\section{RESULTS}

The time interval between alleged or reported drug ingestion and drug screening was $<60$ minutes in 71 cases $(22.9 \%)$, between 60 and 120 minutes in 44 cases $(14.2 \%),>120$ minutes in 125 cases $(40.3 \%)$, unspecified in 70 cases $(22.6 \%)$. The distribution was not different in relation to the results of the screening $\left(\chi^{2}=2.249, \mathrm{p}=0.522\right)$.

\begin{tabular}{llc}
$\begin{array}{l}\text { Table } 1 \text { Characteristics of patients submitted to drug screening for suspected drug } \\
\text { overdose }\end{array}$ & Cases $(\mathbf{n}=310)$ & $\%$ \\
\hline Patient characteristics & & \\
\hline Sex & 147 & 47.4 \\
Men & & \\
Age $(\mathbf{y})$ & 6 & 1.9 \\
$0-10$ & 37 & 11.9 \\
$11-20$ & 89 & 28.7 \\
$21-30$ & 80 & 25.8 \\
$31-40$ & 41 & 13.2 \\
$41-50$ & 14 & 4.5 \\
$51-60$ & 41 & 13.2 \\
$>60$ & 81 & 26.1 \\
Comorbidity & 39 & 12.6 \\
Depression & 32 & 10.3 \\
Anxiety & 18 & 5.8 \\
Psychosis & 12 & 3.9 \\
Neurological diseases & 42 & 13.5 \\
Long term alcohol misuse & 12 & 3.9 \\
Long term drug misuse & & \\
Other diseases & & \\
\hline
\end{tabular}


Table 2 Combined HPLC-immunoenzymatic multidrug screening for suspected drug overdose in patients with specific symptoms related to drug ingestion and in patients without significant symptoms for self poisoning. Blood alcohol measurement is also reported

\begin{tabular}{lll}
\hline Screening procedures & $\begin{array}{l}\text { Symptomatic } \\
\mathbf{n = 2 1 3}(\%)\end{array}$ & $\begin{array}{l}\text { Asymptomatic } \\
\mathbf{n = 9 7}(\%)\end{array}$ \\
\hline REMEDi (urine samples) & $213(100)$ & $97(100)$ \\
REMEDi (serum) & $87(40.8)$ & $30(30.9)$ \\
FPIA (serum) & $79(37.1)$ & $27(27.8)$ \\
FPIA (serum) $\geqslant 2$ & $23(10.8)$ & $7(11.3)$ \\
FPIA (urine samples) & $49(23.0)$ & $2(2.1)$ \\
FPIA (urine samples) $\geqslant 2$ & $9(4.3)$ & $49(50.5)$ \\
Blood alcohol concentration & $200(93.9)$ & \\
\hline
\end{tabular}

\section{Acute intoxication}

In 199 of 310 cases $(64.2 \%)$ the diagnosis was acute overdose on the basis of a concentration of drugs or their metabolites in plasma or urine in amounts to explain primary related symptoms. In 129 cases (41.6) a single drug was responsible for acute intoxication, whereas in $70(22.6 \%)$ two or more drugs were found and in 12 cases $(3.9 \%$ of cases) three or more drugs were present (table 3 and 4 ). In addition, in several positive cases other unsuspected drugs were detected at low concentrations, without relevance for overall clinical management.

There were large differences between the suspected and final diagnosis (table 5). Of the 443 drugs suspected by the clinicians to be involved, only 197 were confirmed. Conversely unsuspected drugs were found in 86 patients. The most common pharmaceutical agents (BZD, other hypnotics, neuroleptics, tricyclic antidepressants, SSRI, other antidepressant) were frequently suspected, and their presence was confirmed in about $20 \%-60 \%$ of cases (table 5 ). In contrast, less common drugs were rarely suspected and only diagnosed by biochemical analysis. Drugs of misuse were only suspected in 19 patients but found in 82 and in 41 patients this was the only drug. If FPIA was the only analysis used, a diagnosis of acute intoxication with confirmatory laboratory results would have been obtained only in 67 of 199 cases $(33.7 \%)$, because of the limited number of drugs detectable by the FPIA method.

On the basis of clinical course and laboratory results, 39 symptomatic patients $(23.9 \%)$ were safely discharged after an observation of three to six hours, and followed up as outpatients. These subjects were intoxicated by short acting, non-life threatening drugs, and had low plasma concentrations and high urinary levels.

\section{Non-toxic concentrations}

In 43 cases $(13.9 \%)$, the concentration of alleged or reported drug was measurable, but at trace, non-toxic concentrations. The most common drugs in this group were BZD in 30 cases, neuroleptics ( 8 cases), tricyclic antidepressant ( 3 cases), SSRI (4 cases), anticonvulsants ( 13 cases), digoxin (3 cases). A diagnosis of acute intoxication would theoretically be excluded with FPIA performed on the basis of suspected

Table 3 Report on drugs responsible for acute intoxication as single agents or in association with other drugs in patients admitted to the ED for self poisoning

\begin{tabular}{|c|c|c|c|}
\hline Drug & $\begin{array}{l}\text { Number of } \\
\text { cases }\end{array}$ & Single agent & Associations (one or more) \\
\hline Benzodiazepines & 71 & 36 & $\begin{array}{l}\text { BZD 3, neuroleptics } 7 \text {, TCA 6, SSRI 1, other antidepressant } \\
6 \text {, anticonvulsants } 2 \text {, morphine } 4 \text {, cocaine } 4 \text {, cannabinoids } \\
2 \text {, fenproporex 1, CC blockers 2, antihistamines 3, } \\
\text { pentoxiphylline 1 }\end{array}$ \\
\hline Other hypnotics & 5 & 4 & Neuroleptics 1 \\
\hline Neuroleptics & 26 & 9 & $\begin{array}{l}\text { BZD 6, neuroleptics 2, TCA 1, SSRI 1, trazodone 1, } \\
\text { anticonvulsants } 1 \text {, antiparkinsonians } 3 \text {, morphine } 3 \text {, } \\
\text { cocaine } 3 \text {, cannabinoids } 1, \beta \text { blockers } 1 \text {, analgesics } 2 \text {, other } \\
\text { hypnotics } 1\end{array}$ \\
\hline $\begin{array}{l}\text { Tricyclic } \\
\text { antidepressants }\end{array}$ & 18 & 9 & BZD 6, neuroleptics 2, SSRI 1, CC blockers 1, cocaine 1 \\
\hline SSRI & 7 & 3 & BZD 1, neuroleptics 1, TCA 1, risperidone 1 \\
\hline $\begin{array}{l}\text { Other } \\
\text { antidepressant }\end{array}$ & 13 & 6 & BZD 6, neuroleptics 1, Antihistamines 1, venlafaxine 1 \\
\hline Anticonvulsants & 7 & 3 & BZD 2, neuroleptics 1, $\beta$ blockers 2 \\
\hline Antiparkinsonians & 7 & 3 & neuroleptics 3, SSRI 1, morphine 1, digoxine 1 \\
\hline CC blockers & 7 & 2 & BZD 1, TCA 1, clorochine 1 \\
\hline$\beta$ blockers & 2 & - & Neuroleptics 1, anticonvulsants 2 \\
\hline Antihistamines & 3 & - & BZD 3, trazodone 1 \\
\hline Analgesics & 6 & 4 & Neuroleptics 2, analgesics 1 \\
\hline Digoxin & 2 & 1 & Antiparkinsonians 1 \\
\hline Other drugs* & 12 & 8 & \\
\hline
\end{tabular}

BZD, benzodiazepines; TCA, tricyclic antidepressants; SSRI, serotonin specific reuptake inhibitors; CC blockers, calcium channel blockers. *They include codeine, scopolamine, roxitromycin, idrossibutirric acid, levodropropizine, disulfiram, pentoxifylline, and tinirazine as single agents and the following associations: dihydroergotamine and morphine, pentoxifylline and BZD, trimethoprim and sulfamethoxazole, dihydroergotamine and oxymetazoline. 
Table 4 Report on drugs of misuse responsible for acute intoxication as single agents or in association with other drugs in patients admitted to the ED for self poisoning

\begin{tabular}{|c|c|c|c|}
\hline Drug of misuse & $\begin{array}{l}\text { Number of } \\
\text { cases }\end{array}$ & Single agent & Associations \\
\hline Morphine & 46 & 30 & $\begin{array}{l}\text { BZD 4, neuroleptics 5, SSRI 1, antiparkinsonians } 1 \text {, cocaine } \\
\text { 10, amphetamines 1, cannabinoids } 1\end{array}$ \\
\hline Cocaine & 24 & 6 & $\begin{array}{l}\text { BZD 4, neuroleptics 3, morphine 9, amphetamines 2, } \\
\text { cannabinoids } 3\end{array}$ \\
\hline Cannabis & 8 & 3 & BZD 2, neuroleptics 2, cocaine 3 \\
\hline MDMA (Ecstasy) & 2 & 2 & \\
\hline Amphetamines & 4 & & BZD 1 , cocaine 2 , morphine 1 \\
\hline
\end{tabular}

MDMA, 3,4 methylenedioxymethamphetamine. Other abbreviations as for table 3.

drugs in only 23 of 43 cases (53.5\%). However, in seven cases trace amounts of drugs, non-indicative of acute intoxication, were detected at first screening, but a second test after admission led to diagnosis of acute intoxication. This was the case of a patient with suspected paracetamol intoxication, initially seen at therapeutic concentrations, of four cases of isolated long acting BZD poisoning, and cases of pentoxyfilline and brotizolam overdose.

\section{Negative screening}

In 68 patients $(21.9 \%$ of total cases) the test did not show the presence of any drug at urinary Remedi screening. Thirty five patients $(51.4 \%)$ had minimal or no symptoms, together with a self harm behaviour, and were reclassified as psychiatric disturbances.

The remaining symptomatic patients had either a blood alcohol concentration in the toxic range $(>1.0 \mathrm{~g} / \mathrm{l})$, and their symptoms were related exclusively to an acute alcohol intoxication ( 11 cases, $16.2 \%$ ), or to a neuropsychiatric disease without evidence of drug intoxication (22 cases, $32.4 \%)$.

Fifteen symptomatic patients of $33(45.5 \%)$ were discharged after an observation period of three to six hours in the ED to recover from mild depression of the central nervous system. They did not re-attend for secondary clinical complications after discharge at a follow up of 30 days.

In a single patient, admitted because of alleged long acting BZD overdose, the initial multidrug screening did not detect any drug at admission. He was admitted because of rapid clinical deterioration and the test was positive at a second assessment, within five hours.

\section{Clinical compared with biochemical diagnosis}

Ingestion of drugs in toxic amounts was clinically suspected in 213 symptomatic patients and in 97 patients suffering from minimum or non-specific symptoms. In only 76 of the 310 patients was the diagnosis fully correct. Symptomatic patients were less likely to have at least one suspected drug not confirmed than asymptomatic patients, whereas they were more likely to have at least one clinically unsuspected drug reported (table 6). In 15 patients (4.8\% of total cases), who had no symptoms when first seen in ED, the diagnosis of drug overdose was possible because at least one unsuspected drug was found at analysis. All these substances would not be detectable by FPIA in plasma or urine specimens, and 13 of 15 cases (excluding one intoxication from selegiline and one from cannabis) were admitted to a general ward for observation.

Twenty nine patients (9.3\%) attended the ED for a suspected poisoning of an unspecified drug (table 6). In 21 of 29 cases $(72.4 \%)$ the screening did not reveal any substance; in one case non-significant concentrations of lamotrigine and clozapine were detected, and in one patient clomipramine and delorazepam was found. In only 6 of 29 cases $(20.7 \%)$ the diagnosis was acute intoxication, a figure significantly lower than that observed in patients attending the ED with the suspicion of a specific drug overdose $\left(\chi^{2}\right.$ value $=24.9 ; \mathrm{p}<0.001)$.

Table 5 Patients with diagnosis of drug overdose and alcohol intoxication in relation to suspected overdose in patients admitted to the ED for intentional self poisoning

\begin{tabular}{lllll}
\hline Drug & Suspected overdose & Diagnosis of overdose & Alcohol intoxication & Drug overdose+alcohol \\
\hline Benzodiazepines & 182 & $71(39.0)$ & $38(29.9)$ & $7(3.8)$ \\
Other hypnotics & 9 & $5(55.6)$ & $2(2.2)$ & - \\
Neuroleptics & 58 & $26(44.8)$ & $21(36.2)$ & $5(8.6)$ \\
Tricyclic antidepressants & 49 & $18(36.7)$ & $11(22.4)$ & $1(2.0)$ \\
SSRI & 22 & $7(31.8)$ & $4(18.2)$ & $1(4.5)$ \\
Other antidepressant & 22 & $13(59.1)$ & $6(27.3)$ & $3(13.6)$ \\
Anticonvulsants & 31 & $7(22.6)$ & $9(29.0)$ & - \\
Antiparkinsonians & 5 & $7(140.0)$ & $1(20.0)$ & - \\
CC blockers & 5 & $7(140.0)$ & - & - \\
B blockers & 5 & $2(40.0)$ & $2(40.0)$ & - \\
Antihistamines & 2 & $3(150.0)$ & - & - \\
Analgesics & 15 & $6(40.0)$ & $2(13.3)$ & - \\
Digoxin & 2 & $2(100.0)$ & - & - \\
Other drugs* & 18 & $12(66.7)$ & $1(5.6)$ & $11(91.7)$ \\
Drugs of misuse & 12 & $46(383.3)$ & $12(100.0)$ & $7(175.0)$ \\
Morphine & 4 & $24(600.0)$ & - & $3(150.0)$ \\
Cocaine & 2 & $8(400.0)$ & $2(100.0)$ & - \\
Cannabis & - & 2 & 1 & - \\
MDMA (Ecstasy) & 1 & $4(400.0)$ & - & \\
Amphetamines & & & \\
\hline Percentages shown in parentheses. & & &
\end{tabular}


Table 6 Pattern of discrepancies between suspected or unsuspected drugs self poisoning in symptomatic and asymptomatic patients

\begin{tabular}{|c|c|c|c|c|}
\hline & $\begin{array}{l}\text { Symptomatic } \\
n=213(\%)\end{array}$ & $\begin{array}{l}\text { Asymptomatic } \\
n=97(\%)\end{array}$ & $\begin{array}{l}\text { Odds ratio } \\
(95 \% \mathrm{Cl})\end{array}$ & p Value \\
\hline Suspected drugs totally confirmed & $60(28.2)$ & $16(16.5)$ & $1.11(0.85$ to 1.45$)$ & 0.428 \\
\hline At least one suspected drug not confirmed & $63(29.6)$ & $56(57.7)$ & $0.62(0.50$ to 0.76$)$ & $<0.001$ \\
\hline At least one unsuspected drug & $37(17.4)$ & $3(3.1)$ & $1.90(1.21$ to 3.00$)$ & 0.005 \\
\hline $\begin{array}{l}\text { At least one suspected drug not confirmed plus } \\
\text { one unsuspected drug }\end{array}$ & $34(16.0)$ & $12(12.4)$ & $0.97(0.72$ to 1.30$)$ & 0.820 \\
\hline Unspecified & $19(8.9)$ & $10(10.3)$ & $0.79(0.57$ to 1.10$)$ & 0.170 \\
\hline
\end{tabular}

In 11 patients $(37.9 \%)$ an acute alcohol intoxication was shown.

\section{DISCUSSION}

This retrospective study suggests that in patients attending an ED for alleged drug poisoning or acute intoxication from drugs of misuse the suspected drugs are not always confirmed in a large proportion of cases. Unsuspected drugs, often drugs of misuse were also detected in large numbers of patients. This is true in both symptomatic patients, where the alleged drug may be more easily suspected, and in asymptomatic cases, where unsuspected drugs, potentially harmful, may be detected by a comprehensive drug screening.

The importance of the multidrug analysis in the management of patient with acute drug overdose has not been proved. Several authors claim that toxicology screening is useful for the treatment of their patients, but only few studies provide quantitative evidence to support this conclusion. ${ }^{13}{ }^{14}$ Other studies suggest that unexpected laboratory findings scarcely influence diagnosis of patients with drug overdose, except for salicylates, iron, acetaminophen. ${ }^{27815}$ The clinical course remains the leading criterion for treatment, but a comprehensive drug screening might be useful in patients with changed mental status, or coma of unclear aetiology for diagnostic purposes, and to exclude unsuspected drugs.

In asymptomatic or uncomplicated patients with confirmed overdose the laboratory screening is unlikely to contribute to management. These subjects have a favourable outcome with minimum intervention, the clinical course might be predictable on the basis of the first few hours of observation, and their conditions are unlikely to deteriorate. ${ }^{16}$ Exceptions may be patients who ingested life threatening doses of drugs with delayed adverse consequences, where an accurate drug identification is mandatory for appropriate decontamination procedures, ${ }^{17}$ or the ingestion of large amounts of long acting, retard formulations as illustrated by the case of the long acting benzodiazipine in this study.

There are differences between clinical judgment and laboratory results, but studies are usually carried out by means of specific drug panels, selected on the basis of a clinical approach. ${ }^{18}$ In our study we used a modified version of a comprehensive drug identification system, which tests over 900 drugs in blood and urine..$^{9-12}$ The advantage of our system is the rapid measurement (available in a turnaround of 60 minutes) of most drugs and their metabolites, which permits the identification of the $\operatorname{drug}(\mathrm{s})$, even when they are almost completely cleared from blood. This may be important to the emergency physician to support the diagnosis of poisoning in a condition where the history referred by patients, relatives, or bystanders is frequently unreliable, the suspicion of drug overdose might simply be raised by the occasional finding of empty drug containers, and symptoms may be non-specific.
Errors in comprehensive screening may lead to both false positive and false negative results. ${ }^{19} 20$ The laboratory finding of a clinically unsuspected drug is not necessary a marker of severity of illness and does not on its own identify patients requiring a closer observation. Our data show that in 43 cases (13.9\%) one or more drugs were measurable in trace quantity without any clinical relevance, and the diagnosis of acute intoxication was made in only seven of these patients, after repeated testing based on the evidence of a worsening clinical course. To avoid any misinterpretation, the results of individual tests need to be always discussed between the analyst and the physician, and compared with clinical status.

The advantages and the limits of the test are different in relation to clinical status. In symptomatic patients, most symptoms are non-specific and shared between drugs. In most of these cases, the comprehensive screening identified one or more harmful substance(s) adding to those clinically suspected, as frequently reported. ${ }^{1}$ Apart from its value for diagnostic purposes, it is not possible to comment on the usefulness of this information for clinical management in a retrospective series. However, there is no doubt about the advantage of a negative test, and particularly of the benefit of a rapid piece of information for decision making. A few patients might be mimicking symptoms, and, according to our policy, nearly $25 \%$ of patients with a negative test were safely discharged with minimum observation after the results were available. The remaining patients were re-evaluated in search of different causes for their symptoms.

In asymptomatic patients with alleged drug poisoning the clinical value of the screening is proved. Over $50 \%$ of cases had a completely negative test but the remaining had an acute intoxication, possibly in a pre-clinical stage. These patients include not only subjects with suspected drugs totally confirmed, but also patients with at least one unsuspected drug with/without a suspected drug not confirmed (15 cases). These patients are a special challenge for emergency physicians, being at risk of errors in diagnosis. In our series, all these patients were admitted to hospital, and most of them developed low grade intoxication symptoms during follow up, and recovered uneventfully.

A few limitations of our multidrug screening policy should be discussed. Firstly, our ED is part of a community hospital, where patients are treated for at least two to four hours before admission. Our results may not applicable to different hospital, where patients are moved out of the ED more quickly.

Secondly, a diagnosis of multidrug overdose may generate unnecessary admissions to an ICU, independently of the clinical condition of poisoned patients. Over-admission is considered a less serious error than lack of admission of a patient who could have benefited by ICU treatment. Some patients might also be admitted for brief surveillance because a possible suicidal behaviour, independently of the need for specific therapeutic procedures. ${ }^{16}$ In our setting 26 of 32 cases 
(81.2\%) were admitted to ICU for only 24 hours, and up to $50 \%$ of them did not have to any specific therapeutic measure. Over-admission might be compared against the advantage of safe rapid discharge in patients with negative tests. The cost/benefit of our policy might be tested in a prospective series considering the total cost of the screening, including salaries and reagents (in our hospital about $€ 120$ for patients with a negative test and $€ 150$ for positive analyses).

Finally, although the library of detectable drugs is periodically upgraded (by December 2001 the number of detectable drugs has increased to 1100), it must be kept in mind that this will never bring the system to $100 \%$ sensitivity.

In conclusion, our integrated system for a rapid comprehensive screening of acute drug poisoning adds to the diagnosis of patients with suspected drug poisoning, mainly identifying unsuspected drugs in symptomatic patients and excluding purported drugs in asymptomatic subjects. It might also be helpful to predict the clinical course in selected cases, making the clinical observation more accurate. Its value needs to be tested in a prospective study.

\section{Contributors}

Andrea Fabbri conceived the study, wrote the protocol, coordinated the data collection, interpretation of results, and wrote the paper. Giulio Marchesini, contributed to study design, carried out statistical analyses, interpretation of the results, and cowrote the paper. Saverio Ruggeri carried out laboratory analyses and critical review of the paper. Alberto Vandelli contributed to study design, study coordination, interpretation of the results, and critical review of the paper. All authors approved the final version of the paper.

\section{ACKNOWLEDGEMENTS}

We are grateful to the emergency medical system personnel for helpful support. We thank the other members of the Research Group: R Azzali, G Bertozzi, M Dente, M Focacci, N Fuzzi, S Galanti, I Giondi, F Grilli, T Iervese, L Leonardi, M Maroni, G Martino, G Righetti, F Rossi, M Spada, R Todeschini, M Tonini, for data collection and participation in the study.

\section{Authors' affiliations}

A Fabbri, A Vandelli, Dipartimento Emergenza-Urgenza Accettazione, Ospedale GB Morgagni, Azienda Unità Sanitaria Locale di Forli, Italy S Ruggeri, Laboratorio di Patologia Clinica, Ospedale GB Morgagni
G Marchesini, Dipartimento di Medicina Interna e Gastroenterologia, Servizio di Malattie del Metabolismo, Università degli Studi di Bologna, Italy

\section{REFERENCES}

1 Brett AS. Implications of discordance between clinical impression and toxicology analysis in drug overdose. Arch Intern Med 1988;148:437-41.

$2 \mathrm{McC}$ oy DJ, Trestrail JH 3rd. Findings of ten years of clinical drug screening. Vet Hum Toxicol 1988;30:34-8.

3 Taylor RL, Cohan SL, White JD. Comprehensive toxicology screening in the emergency department: an aid to clinical diagnosis. Am J Emerg Med 1985:3:507-11.

4 Osterloh JD. Utility and reliability of emergency toxicologic testing. Emerg Med Clin North Am 1990;3:693-723.

5 Durback LF, Scharman EJ, Brown BS. Emergency physicians perception of drug screens at their hospitals. Vet Hum Toxicol 1998;40:234-7.

6 Mahoney JD, Gross PL, Stern TA, et al. Quantitative serum toxic screening in the management of suspected drug overdose. Am J Emerg Med 1990;8:16-22.

7 Bailey DN. Results of limited versus comprehensive toxicology screening in a university medical center. Am J Clin Pathol 1996;105:572-5.

8 Skelton H, Dann LM, Ong RT, et al. Drug screening of patients who deliberately harm themselves admitted to the emergency department. Ther Drug Monit 1998;20:98-103.

9 Sadeg N, Francois G, Petit B, et al. Automated liquid-chromatographic analyzer used for toxicology screening in a general hospital: 12 months' experience. Clin Chem 1997;43:498-504.

10 Baskin LB, Morgan DL. Drugs detected in patients suspected of acute intoxication. Tex Med 1997:93:50-8.

11 Pechard A, Besson AS, Mialon A, et al. Critical analysis of different methods used for toxicology screening in emergency laboratory. Ann Biol Clin 1999;57:525-37.

12 Ohtsuji M, Lai JS, Binder SR, et al. Use of REMEDi HS in emergency toxicology for a rapid estimate of drug concentration in urine, serum, and gastric samples. J Forensic Sci 1996;41:881-6.

13 Wolff K, Farrell M, Marsden J, et al. A review of biological indicators of illicit drug use, practical consideration and clinical usefulness. Addiction 1999:94:1279-98.

14 Perrone J, De Roos F, Jayaraman S, et al. Drug screening versus history in detection of substance use in ED psychiatric patients. Am J Emerg Med 2001; 19:49-51.

15 Belson MG, Simon HK, Sullivan K, et al. The utility of toxicologic analysis in children with suspected ingestions. Pediatr Emerg Care 1999;15:383-7.

16 Brett AS, Rothschild N, Gray R, et al. Predicting the clinical course of intentional drug overdose. Implications for use of the intensive care unit. Arch Intern Med 1987; 147:133-7.

17 AACT/EAPCCT. Position statements on gastrointestinal decontamination. Clin Toxicol 1997:35:695-762.

18 Kellermann AL, Fihn SD, Lo Gerfo JP, et al. Impact of drug screening in suspected overdose. Ann Emerg Med 1987;16:1206-16.

19 Ingelfinger JA, Isakson BA, Shine D, et al. Reliability of the toxic screen in drug overdose. Clin Pharmacol Ther 1981;29:570-5.

20 Hansen HJ, Caudill SP, Boone DJ. Crisis in drug testing: fesults of CDC blind study. JAMA 1985;253:2382-7. 